\title{
Survival rates of cancer patients with and without rheumatic disease: a retrospective cohort analysis
}

\author{
Jin Kyun Park ${ }^{1 \dagger}$, Ji Ae Yang ${ }^{1 \dagger}$, Eun Young Ahn', Sung Hae Chang ${ }^{2}$, Yeong Wook Song ${ }^{1}$, Jeffrey R. Curtis ${ }^{3}$ \\ and Eun Bong Lee ${ }^{1 *}$
}

\begin{abstract}
Background: To compare the outcomes of gastric, colon, lung, and breast cancer patients with and without rheumatic diseases (RD).

Methods: This retrospective study compared the cancer survival rates of a cohort of 122 cancer patients with rheumatoid arthritis (RA), systemic lupus erythematosus (SLE), dermatomyositis/polymyositis (DM/PM), or systemic sclerosis with that of a cohort of 366 age-, sex-, and, cancer type-matched patients without RD who received medical care from 2000 to 2014. Staging, comorbidities, and functional status were ascertained. Survival was compared using the Kaplan-Meier method. Relative risk of death was estimated as a hazard ratio (HR) using Cox regression analysis.
\end{abstract}

Results: The mean age of the RD patients at the time of cancer diagnosis was $58.7 \pm 11.5$ years. The overall survival rate of gastric cancer patients did not differ between the cohorts. The survival of lung or breast cancer was worse in patients with RA or DM/PM than in those without RD (all, $p<0.05$ ). After adjusting for cancer stage, comorbidity index, performance status and age at the time of cancer diagnosis (as well as interstitial lung disease for lung cancer group), the mortality rate among lung cancer patients with RA was significantly higher $(\mathrm{HR}, 1.81 ; 95 \% \mathrm{Cl}, 1$. 03-3.18) than that of lung cancer patients without RD, whereas SSC was associated with decreased mortality of lung cancer ( $\mathrm{HR}, 0.16 ; 95 \% \mathrm{Cl}, 0.04-0.58)$. DM/PM were associated with increased mortality of breast cancer patients (HR, 297.39; 95 \% Cl, 4.24-20842.33).

Conclusions: RA and DM/PM seemed to be associated with a higher mortality in patients with lung or breast cancers, whereas SSc seemed to be associated with decreased mortality in patients with lung cancer. It is warranted to explore the survival effect of tailored cancer treatments according to specific RD.

Keywords: Rheumatic diseases, Cancer, Staging, Mortality, Survival

\section{Background}

Rheumatic disease (RD) is characterized by chronic systemic inflammation involving multiple organs. Unopposed inflammation and the production of cytokines, growth hormones, and toxic reactive oxygen species may promote the progression of precancerous cells into a clinically significant cancer [1]. Impaired immune surveillance

\footnotetext{
*Correspondence: leb7616@snu.ac.kr

${ }^{\dagger}$ Equal contributors

${ }^{1}$ Division of Rheumatology, Department of Internal Medicine, Seoul National University College of Medicine, 101, Daehak-ro, Jongno-gu, Seoul 03080, Korea

Full list of author information is available at the end of the article
}

associated with underlying $\mathrm{RD}$ and long-term immunosuppressive therapy might not only promote the survival and proliferation of cancer cells, but also increase the risk of infection, a main cause of death during cancer treatment [2-4]. In short, cancer patients with RD may have a worse prognosis than those without RD. Conversely, cytotoxic autoimmune cells in patients with $\mathrm{RD}$ might eliminate cancer cells better. Also, the prolonged use of non-steroidal anti-inflammatory drugs (NSAIDs) and antimetabolites such as methotrexate might inhibit proliferative stimuli, thereby inhibiting cancer development and progression [5, 6]. Furthermore, the close medical monitoring 
received by $\mathrm{RD}$ patients during longitudinal follow up might lead to early detection of cancers and more prompt treatment, ultimately resulting in a better outcome [7, 8].

Although a solid body of evidence supports a close association between RD and incident cancers, data regarding outcomes for RD patients who develop incident cancer are scarce and often conflicting $[9,10]$. We previously reported that the cancer outcome in patients with Takayasu arteritis was excellent, suggesting that the underlying autoimmune disease might not negatively influence cancer outcome, at least for certain RDs [11]. Here, we aimed to investigate whether cancer patients with specific RDs have a worse outcome than those without RDs by comparing the survival of RD patients with four common cancers (i.e., gastric, colon, lung, and breast) with that of matched cancer patients without RDs.

\section{Methods}

\section{Patients}

The medical records of patients with rheumatoid arthritis (RA), systemic lupus erythematosus (SLE), systemic sclerosis (SSc), and dermatomyositis/polymyositis (DM/PM) that received longitudinal clinical care at Seoul National University Hospital from January 2000 to April 2014 were retrospectively reviewed, and those that had developed a gastric, colon, lung, or breast cancer after their RD diagnosis were enrolled in the study (these patients comprised the RD-exposed cancer cohort). The patients in whom cancer was diagnosed before RD were excluded, since the aim of the study was to investigate the effects of RD on the cancer outcome. RA, SLE, SSc and PM/DM were diagnosed according to the 1987 revised classification criteria of the American College of Rheumatology (ACR) for RA, the 1997 ACR classification criteria for SLE, the 1980 preliminary ACR classification criteria for SSc, and the Bohan and Peter criteria for PM or DM, respectively [12-15]. The non-RD-exposed cancer cohort comprised 366 age-, sex-, and cancer site-matched patients without RD who were diagnosed with cancer in the same year as the RD patients and who received medical care at the same hospital. These patients were randomly selected from the medical record archive so as to achieve a matching ratio of 1 to 3 for each RD. The study was approved by the Institutional Review Board of the Seoul National University Hospital. The need for patient consent was waived by the Review Board as the study involved minimal risk and its retrospective nature meant that no identifiable information was used.

\section{Cancer assessment}

A complete data set for each cancer, including histology, stage, and treatment, was obtained. All cancer cases were diagnosed histopathologically and confirmed by biopsy. Cancers were staged according to the American
Joint Committee on Cancer (AJCC) 7th staging system. The Eastern Cooperative Oncology Group performance status (ECOG-PS) and the Charlson comorbidity index at the time of cancer diagnosis were estimated after reviewing electronic medical records. An ECOG-PS of 0 indicates that the patient is asymptomatic and a score of 1 indicates that patient is restricted in terms of strenuous activity but is able to ambulate and carry out light work; however, an ECOG-PS $\geq 2$ indicates a significant limitation in performance status [16]. The Charlson comorbidity index predicts 10 -year mortality for a patient who may have a range of 22 comorbid conditions, including cardiovascular disease and lung disease [17]. The index sums the assigned scores for each comorbid condition, e.g., connective tissue disease is assigned a score of 1 , while a malignant tumor is assigned a score of 6 . In this study, the scores for connective tissue disease and cancer were omitted from the Charlson comorbidity index.

\section{Statistical analysis}

Student t-tests or analysis of variance (ANOVA) were used to compare continuous variables between groups and the Chi-square or Fisher's exact test were used to compare categorical variables. Survival curves were generated using the Kaplan-Meier method. Differences in survival were compared using the log-ranks test. Follow up began at the time of cancer diagnosis and was censored at the time of death or on the last day on which survival status was followed up, whichever came first. The survival status of the all patients was ascertained using the National Death Register of Statistics Korea (www.kostat.go.kr). The relative risk of death was estimated using Cox's proportional hazard ratio (HR) for survival by adjusting for stage, ECOG performance status, Charlson comorbidity index, and age in years at the time of cancer diagnosis. HR for lung cancer mortality was adjusted for the interstitial lung disease (ILD) status as well. $P \leq 0.05$ was considered significant. All analyses were performed using IBM SPSS (statistics version 19.0, Chicago, IL, USA).

\section{Results}

Demographic characteristics of RD patients with cancer During the follow-up period, 122 RD patients with one of the four selected types of incident cancer were identified. RA was the most common RD (80 patients; $65.6 \%$ ), followed by DM/PM (16 patients; $13.1 \%)$, SSc (13 patients; $10.7 \%$ ), and SLE (13 patients; $10.7 \%)$. The mean age at RD diagnosis was $52.4 \pm 13.5$ years (Table 1 ). Except for the DM/PM group, the number of females was higher than that of males. There were 28 cases of gastric cancer (23.0\%), 23 cases of colon cancer (18.9\%), 44 cases of lung cancer (36.1\%), and 27 cases 
Table 1 Characteristics of 122 patients with rheumatic diseases and 366 cancer-matched controls without RD

\begin{tabular}{|c|c|c|c|c|c|c|}
\hline & RA $(n=80)$ & $\operatorname{SLE}(n=13)$ & $\operatorname{SSC}(n=13)$ & DM/PM $(n=16)$ & All RD $(n=122)$ & Non-RD $(n=366)$ \\
\hline Female, $n(\%)$ & $59(73.8)$ & $13(100.0)$ & $10(76.9)$ & $6(37.5)$ & $88(72.1)$ & $264(72.1)$ \\
\hline Age at RD dx, yrs, & $54.7 \pm 12.8$ & $42.4 \pm 12.9$ & $45.6 \pm 16.1$ & $58.2 \pm 9.9$ & $52.4 \pm 13.5$ & N/A \\
\hline Age at cancer dx, yrs, & $60.2 \pm 11.3$ & $52.2 \pm 9.7$ & $54.2 \pm 13.9$ & $59.7 \pm 9.3$ & $58.7 \pm 11.5$ & $58.6 \pm 11.6$ \\
\hline Follow-up duration, yrs & $14.4 \pm 12.0$ & $17.3 \pm 10.2$ & $10.7 \pm 7.1$ & $13.0 \pm 11.2$ & $12.8 \pm 9.7$ & $12.8 \pm 9.7$ \\
\hline \multicolumn{7}{|l|}{ Cancer type, $n$ (\%) } \\
\hline Stomach & $18(22.2)$ & $4(30.8)$ & $2(15.4)$ & $4(25.0)$ & $28(23.0)$ & $83(22.7)$ \\
\hline Colon & $16(20.0)$ & $3(23.1)$ & $1(7.7)$ & $3(18.8)$ & $23(18.9)$ & $69(18.9)$ \\
\hline Lung & $28(35.0)$ & $0(0)$ & $10(76.9)$ & $6(37.5)$ & $44(36.1)$ & $133(36.3)$ \\
\hline Breast & $18(22.5)$ & $6(46.2)$ & $0(0.0)$ & $3(18.8)$ & $27(22.1)$ & $81(22.1)$ \\
\hline Interstitial lung disease, $n$ (\%) & $6(7.5)$ & $1(7.7)$ & $7(53.8)$ & $3(18.8)$ & $17(13.9)$ & $3(0.8)$ \\
\hline \multicolumn{7}{|l|}{ Treatment of RD, $n(\%)$} \\
\hline Methotrexate & $53(66.3)$ & $1(7.7)$ & $0(0)$ & $5(31.3)$ & $59(48.4)$ & N/A \\
\hline Other DMARDs & $57(71.3)$ & $6(46.2)$ & $2(15.4)$ & $6(37.5)$ & $71(58.2)$ & N/A \\
\hline Corticosteroids & $48(60.0)$ & $7(58.3)$ & $4(30.8)$ & $13(81.3)$ & $72(59.0)$ & N/A \\
\hline TNF inhibitors & $4(5.0)$ & $0(0)$ & $0(0)$ & $0(0)$ & $4(3.3)$ & N/A \\
\hline Others $^{a}$ & $5(6.3)$ & $4(30.8)$ & $2(15.4)$ & $4(25.0)$ & $15(12.3)$ & N/A \\
\hline
\end{tabular}

Data are expressed as the mean \pm SD or as number (\%)

$D x$ diagnosis, DMARD disease modifying antirheumatic drug, $D M / P M$ dermatomyositis/polymyositis, $N$ number, $N / A$ not applicable, $R A$ rheumatoid arthritis, $R D$ rheumatic disease, SLE systemic lupus erythematosus, SSC systemic sclerosis, TNF tumor necrosis factor, Yrs years

athers include tocilizumab, rituximab, abatacept, bucillamine, and cyclosporine

of breast cancer (22.1\%). The mean age of the RD patients at the time of cancer diagnosis was $58.7 \pm$ 11.5 years. The mean time from RD diagnosis to cancer diagnosis was 5.8 [range: 0, 36.3] years. Notably, the mean time between DM/PM and cancer diagnoses was 2.2 [range: $0,8.4$ ] years. The time between the breast cancer and DM/PM diagnosis was relatively longer as compared to gastric, colon and lung cancer (Additional file 1: Table S1).

\section{Characteristics of RD patients with cancer}

The ECOG performance score for RD patients with colon cancer was better than that for their matched non-RD counterparts whereas that for RD patients with lung cancer was worse (Table 2, upper row).

Although the proportion of RD patients with gastric, colon, and lung cancer with a Charlson comorbidity score $\geq 1$ was greater than that of matched cancer patients without RD, the difference was significant for RD patients with colon cancer $(65.2 \%$ for patients with RD vs. $33.3 \%$ for patients without RD; $p=0.025$ ) and those with lung cancer $(50.0 \%$ for patients with RD vs. $32.3 \%$ for patients without RD; $p=0.047$ ) (Table 2, middle row).

Significantly more stage 1 breast cancers were detected in RD patients than in their matched counterparts without RD (51.9 \% vs. $29.6 \%$, respectively; $p=0.036)$. Stage IV lung cancer was more prevalent and stage II less prevalent in RD patients than in their matched counterparts without RD (36.4\% vs. $10.5 \% ; p<0.001$ for stage
IV and 11.4\% vs. $57.9 \% ; p<0.001$ for stage II) (Table 2, bottom rows). Staging of gastric and colon cancers did not differ between the two groups.

\section{Cancer mortality in RD patients versus non-RD patients}

During the follow-up period, 45 (36.9\%) of the 122 patients with RD died. One SSc patient with colon cancer died due to progression of SSc-associated interstitial lung disease (ILD). One RA patient with breast cancer died of congestive heart failure $(n=1)$ and another of old age $(n=1)$. The remaining $42(93.3 \%)$ of 45 deaths occurred due to the cancer progression or complications during cancer treatment (Table 3).

The overall survival of patients with gastric cancer did not differ between the two groups (Fig. 1a). Of those with colon cancer, one patient with SSc had a worse outcome than their matched counterparts without RD (Fig. 1b). Mortality was significantly worse for lung and breast cancer patients with RA or DM/PM than for those without RD (Fig. 1c and d).

Next, the relative risk of $\mathrm{RD}$ with respect to mortality was estimated after adjusting for cancer stage, comorbidity index, performance status, and age at the time of cancer diagnosis. In case of lung cancer, the presence of ILD was also considered for HR calculation. Compared with matched cancer patients without RD, RA was associated with a significant increase in the mortality of lung cancer (HR, 1.81; 95 \% CI, 1.03-3.18 (Fig. 2). DM/PM was associated with a 297.39 -fold increase in mortality 
Table 2 Baseline cancer-related characteristics of the 122 RD patients with cancer and the 366 age-, sex-, and cancer-matched controls without RD

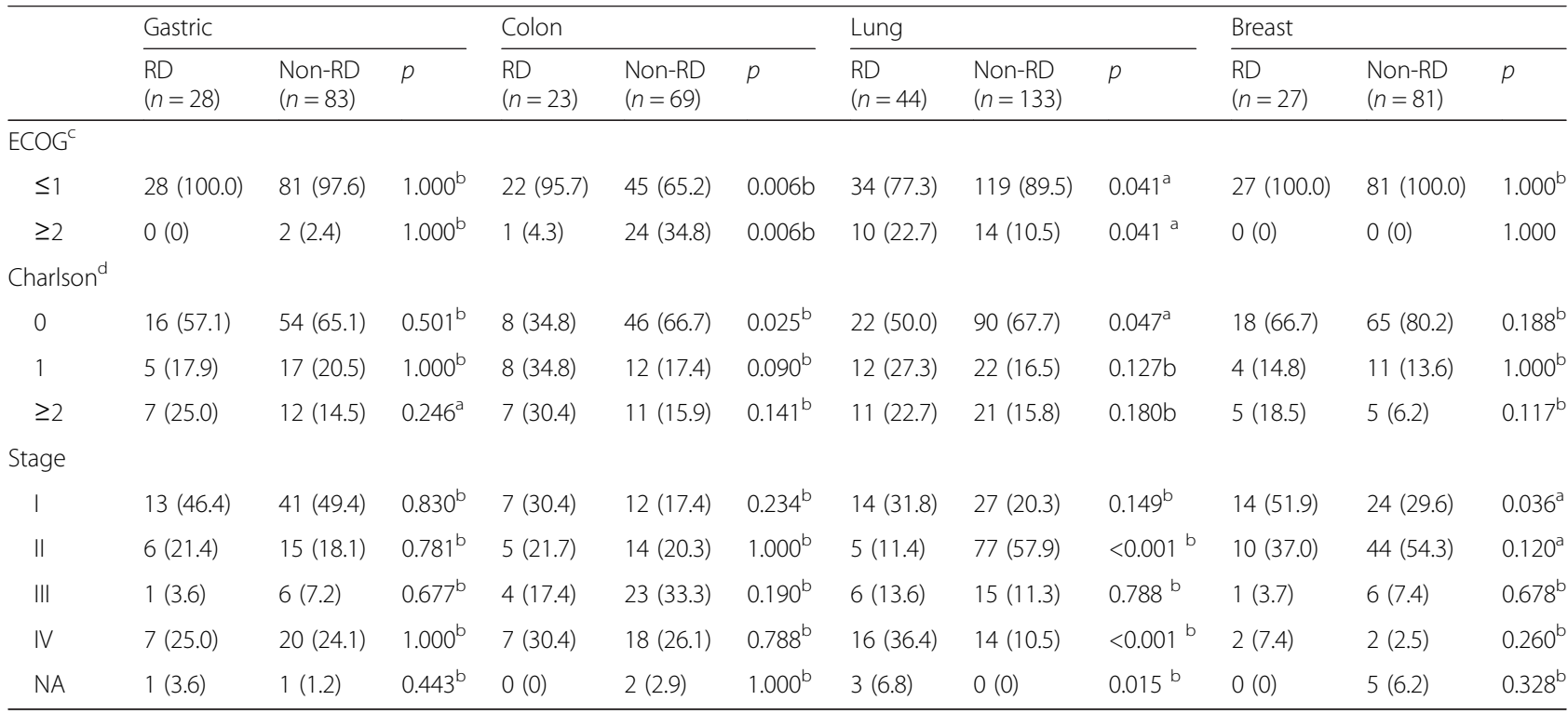

Data are expressed as number (\%)

$F$ female, NA not applicable, $R D$ rheumatic disease

${ }^{\mathrm{a} C h i}$-square test, ${ }^{\mathrm{b}}$ Fisher's exact test

'Eastern Cooperative Oncology Group (ECOG) performance status

${ }^{\mathrm{d}}$ The Charlson comorbidity index was adjusted for connective tissue disease and cancer

for breast cancer (95 \% CI, 4.24-20842.33) patients when compared with matched controls without RD. Strikingly, SSc was associated with decreased mortality for lung cancer (HR 0.16; 95 \% CI, 0.04-0.58).

\section{Discussion}

The present study shows that the mortality associated with breast and lung cancer differs markedly depending on the type of RD: patients with DM/PM with lung cancer had a much worse outcome than counterparts without RD, whereas lung cancer patients with SSc had a better outcome than those without RD.

Although all RDs arise due to defective immune response with subsequent chronic inflammation, the difference in immune dysregulation with preferential production of specific cytokines is associated with varying clinical manifestation [18]. Thus, RDs are classified by the main site of involvement; RA and DM/PM involve primarily joints and muscle, respectively, as the main target organ. SSc is characterized by systemic fibrosis which is considered as an overshooting wound healing $[19,20]$. Inflammation of SLE is mediated by formation and deposition of immune complexes in many tissues, followed by subsequent complement activation leading to organ damage [21]. Thus, the difference in the immune dysregulation and the associated clinical manifestations in individual RDs might influence their cancer incidence and outcome [10].
Cancer survival depends mainly on cancer stage, comorbidities, and functional status at the time of cancer diagnosis, all of which determine the treatment strategy and outcome $[17,22]$. Here, we found that the adjusted Charlson comorbidity index (adjusted for the presence of cancer and connective tissue disease) was worse only for RD patients with colon and lung cancer when compared with matched cancer patients without RD. The ECOG performance status was similar between the RD patients and matched cancer patients without RD (Table 2). This finding suggests that underling RDs might have little influence on performance status and comorbidities in cancer patients.

Since RD patients are followed up more closely during their longitudinal care, we expected that more cancers would be detected at an early stage [7]. However, we found that this was only the case for breast cancer. In Korea, the National Cancer Screening Program (Korean NCSP) recommends that women aged 40 years or over undergo a screening mammogram once every 2 years. The cumulative screening rate for breast cancer in eligible women was reported as high as $77.9 \%$ by 2012 in Korea [23]. One might speculate that their underlying chronic illness makes patients with $\mathrm{RD}$ more vigilant about health issues; thus, they are more likely to follow this recommendation, especially since they have better access to health care services. Strikingly, there was no difference in the stage of gastric cancer between RD and non-RD groups. Chronic use of NSAIDs often leads to 
Table 3 Cause of death of 45 patients with rheumatic disease and cancer

\begin{tabular}{|c|c|c|c|c|c|c|}
\hline & $\mathrm{RD}$ & Cancer & Cause of death & Stage & $\mathrm{ECOG}^{\mathrm{a}}$ & Comorbidity $^{\mathrm{b}}$ \\
\hline 1 & DM/PM & Breast & Cancer progression & 2 & 1 & 1 \\
\hline 2 & DM/PM & Gastric & Cancer progression & 4 & 0 & 0 \\
\hline 3 & DM/PM & Lung & Cancer progression & 1 & 0 & 0 \\
\hline 4 & DM/PM & Lung & Cancer progression & 1 & 0 & 1 \\
\hline 5 & DM/PM & Lung & Cancer progression & 2 & 2 & 1 \\
\hline 6 & DM/PM & Lung & Cancer progression & 2 & 2 & 0 \\
\hline 7 & DM/PM & Lung & Cancer progression & 3 & 2 & 0 \\
\hline 8 & DM/PM & Lung & Cancer progression & NA & 0 & 0 \\
\hline 9 & RA & Breast & Cancer progression & 2 & 0 & 2 \\
\hline 10 & RA & Breast & Cancer progression & 4 & 1 & 0 \\
\hline 11 & RA & Breast & Congestive Heart failure & 1 & 0 & 1 \\
\hline 12 & RA & Breast & Old age & 2 & 0 & 2 \\
\hline 13 & RA & Colon & Cancer progression & 1 & 1 & 1 \\
\hline 14 & RA & Colon & Cancer progression & 3 & 2 & 2 \\
\hline 15 & RA & Colon & Cancer progression & 4 & 1 & 2 \\
\hline 16 & RA & Lung & Cancer progression & 1 & 0 & 0 \\
\hline 17 & RA & Lung & Cancer progression & 1 & 0 & 0 \\
\hline 18 & RA & Lung & Cancer progression & 1 & 0 & 0 \\
\hline 19 & RA & Lung & Cancer progression & 2 & 1 & 2 \\
\hline 20 & RA & Lung & Cancer progression & 2 & 0 & 0 \\
\hline 21 & RA & Lung & Cancer progression & 2 & 0 & 1 \\
\hline 22 & RA & Lung & Cancer progression & 3 & 2 & 1 \\
\hline 23 & RA & Lung & Cancer progression & 3 & 0 & 1 \\
\hline 24 & RA & Lung & Cancer progression & 3 & 0 & 1 \\
\hline 25 & RA & Lung & Cancer progression & 3 & 1 & 2 \\
\hline 26 & RA & Lung & Cancer progression & 4 & 1 & 0 \\
\hline 27 & RA & Lung & Cancer progression & 4 & 2 & 0 \\
\hline 28 & RA & Lung & Cancer progression & 4 & 0 & 0 \\
\hline 29 & RA & Lung & Cancer progression & 4 & 1 & 1 \\
\hline 30 & RA & Lung & Cancer progression & 4 & 2 & 2 \\
\hline 31 & RA & Lung & Cancer progression & 4 & 2 & 1 \\
\hline 32 & RA & Lung & Cancer progression & 4 & 1 & 0 \\
\hline 33 & RA & Lung & Cancer progression & 4 & 2 & 1 \\
\hline 34 & RA & Lung & Cancer progression & 4 & 0 & 0 \\
\hline 35 & RA & Lung & Cancer progression & 4 & 1 & 0 \\
\hline 36 & RA & Lung & Cancer progression & 4 & 0 & 2 \\
\hline 37 & RA & Lung & Cancer progression & NA & 1 & 0 \\
\hline 38 & SLE & Breast & Cancer progression & 4 & 1 & 0 \\
\hline 39 & SLE & Colon & Cancer progression & 1 & 0 & 2 \\
\hline 40 & SSC & Colon & ILD aggrevation & 3 & 1 & 2 \\
\hline 41 & SSC & Lung & Cancer progression & 4 & 1 & 2 \\
\hline 42 & SSC & Lung & Cancer progression & 4 & 2 & 1 \\
\hline
\end{tabular}


Table 3 Cause of death of 45 patients with rheumatic disease and cancer (Continued)

\begin{tabular}{lllllll}
\hline 43 & SSC & Lung & Cancer progression & 4 & 1 & 0 \\
44 & SSC & Lung & Cancer progression & 4 & 0 & 0 \\
45 & SSC & Lung & Cancer progression & NA & 2 & 2 \\
\hline
\end{tabular}

$F$ female, NA not available, $R D$ rheumatic disease, DM dermatomyositis, PM polymyositis, RA rheumatoid arthritis, SLE systemic lupus erythematosus, SSC systemic sclerosis ${ }^{a}$ An Eastern Cooperative Oncology Group (ECOG) performance status

${ }^{\mathrm{b}}$ The Charlson comorbidity index was adjusted for connective tissue disease and cancer

gastrointestinal discomfort; such patients are more likely to undergo esophagogastroduodenoscopy (EGD), which would detect "incidental" cancers at the early stage [24]. In Korea, the high prevalence of Helicobacter pylori and associated gastritis and gastric cancer lower the threshold for EGD, which is readily available at low cost. As for breast cancer, the Korean NCSS offers gastric cancer screening, which includes a double contrast barium upper gastrointestinal series or EGD once every 2 years.
In 2012, the cumulative screening rate was reported to be $77.9 \%$ of the eligible people [23]. The lack of difference in gastric cancer staging between the RD-exposed cohort and the non-RD-exposed cohort may be explained by the high screening rate for gastric cancer in the general population in Korea [25]. Advanced lung cancer was detected more often in patients with RD, consistent with a prior report showing that up to $83 \%$ of lung cancers associated with connective tissue disease a Gastric cancer

Overall $p=0.412$

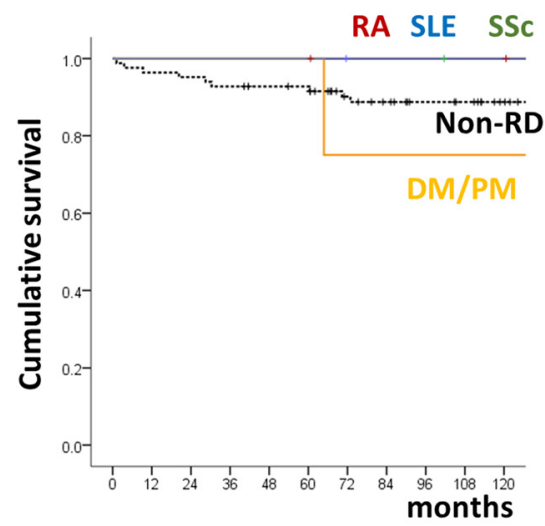

C

Lung cancer

Overall $p=0.002$

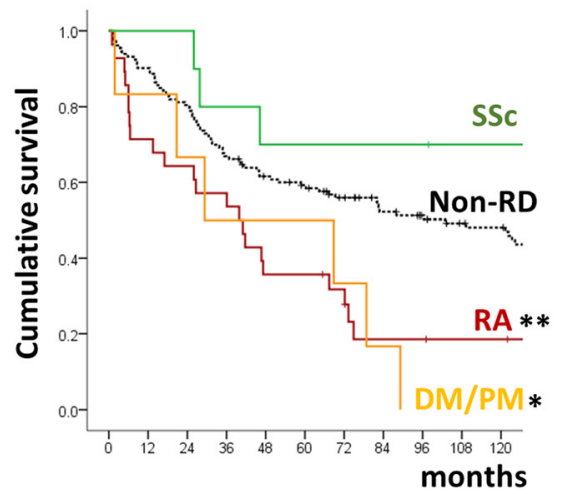

b Colon cancer

Overall $p<0.001$

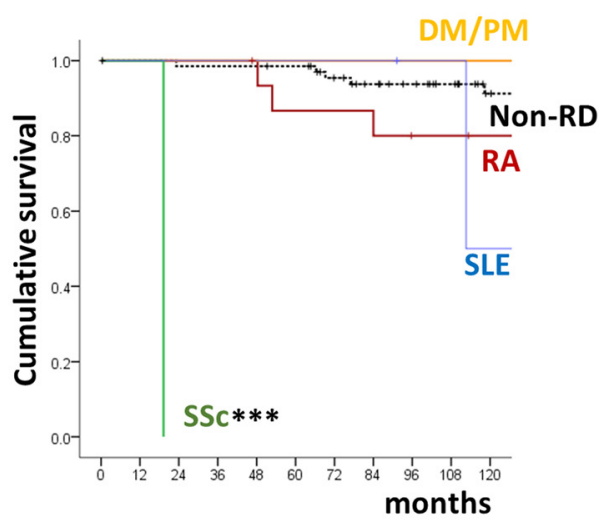

d Breast cancer

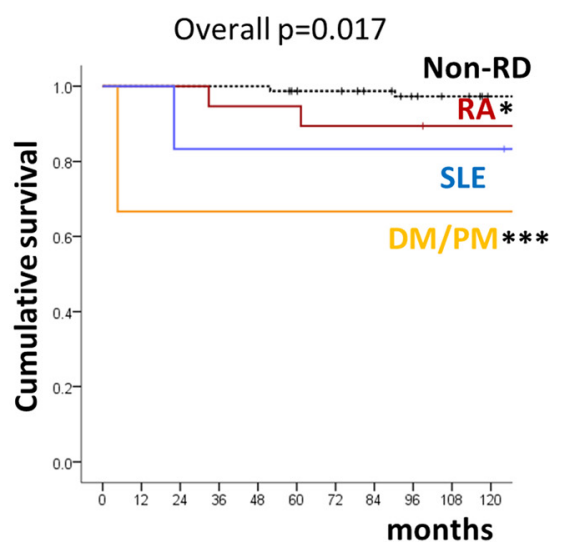

Fig. 1 Kaplan-Meier survival curves for cancer patients with gastric cancer (a), colon cancer (b), lung cancer (c) and breast cancer (d) were compared with those for matched cancer patients without rheumatic diseases. Tick marks indicate censoring events. The $p$ value was calculated using the log rank test. DM, dermatomyositis; ND, not determined; PM, polymyositis; RA, rheumatoid arthritis; RD, rheumatic disease; SLE, systemic lupus erythematosus; SSC, systemic sclerosis. ${ }^{*} p<0.05,{ }^{* *} p<0.01$ and ${ }^{* * *} p<0.001$ compared with the non-RD exposed cancer cohort 

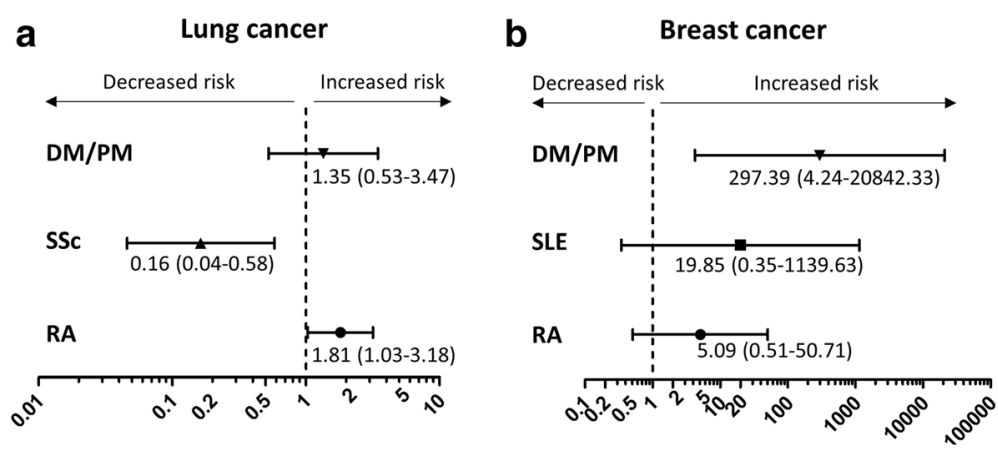

Fig. 2 Hazard ratios (HR) and $95 \%$ confidence intervals for overall survival of patients with lung a or breast b cancer. HR was calculated by adjusting for cancer stage, performance status, comorbidity index, and age at the time of cancer diagnosis. HR for lung cancer was adjusted also for the presence of ILD. ND, not determined; DM, dermatomyositis; PM, polymyositis; RA, rheumatoid arthritis; SLE, systemic lupus erythematosus; SSc, systemic sclerosis

were detected at stage IV [26]. As RD often affects the lung in the form of ILD, the malignant mass might be hidden by diffuse parenchymal changes until it manifests clinically, leading to delayed cancer detection (Table 2) [27]. Alternatively, the defective immune surveillance associated with RD or immunosuppressive treatment facilitates rapid cancer progression. Of note, the Korean NCSS does not recommend screening for lung cancer. Overall, we found the similar (or at least no better) staging of cancer patients with RD and cancer-matched controls without RD in the present study interesting, and it raises questions as to whether patients with $\mathrm{RD}$ might benefit from cancer screening guidelines tailored to their underlying RD. Further studies are needed to compare the cancer screening rates between patients with RD and the general population.

The survival of lung cancer patients with RA or DM/ PM was worse than that for SSc patients and those without RD (Fig. 1c). This is striking, since ILD, which was present in 7 (53.8 \%) SSc patients in the present study, would negatively affect pulmonary function and, therefore, cancer outcome. As shown in Table 1, ILD develops in a relatively low proportion of RA patients and may be clinically asymptomatic [28]. ILD is rare as in $\mathrm{DM} / \mathrm{PM}$ patients with cancer, although it is present in $\geq 60 \% \mathrm{DM} / \mathrm{PM}$ patients in general [29]. After adjusting for cancer stage, comorbidities, performance status, age at the time of cancer diagnosis, and ILD status, we found that RA per se was associated with increased mortality of lung cancer patients by 1.81 -fold ( $95 \mathrm{CI}$, 1.03-3.18), whereas SSc was associated with decreased mortality of lung cancer (HR, 0.16; $95 \% \mathrm{CI}, 0.04-0.58$ ). The results presented herein suggest that the additional presence of ILD is not associated with a worse outcome for lung cancer in patients with SSc. It is tempting to speculate that the accumulation of autoimmune cells associated with ILD provides a harsh environment for cancer cells to grow [30, 31].
The overall mortality rate was high for breast cancer patients with RA or DM/PM (Fig. 1d), when compared with the cancer-matched controls without RD (Table 2, bottom row), even though more breast cancers were diagnosed at stage I. After adjusting for cancer stage, comorbidities, and performance status, DM/PM is associated with higher mortality of breast cancer, similar to a prior report [32]. RA also tended to show a higher death rate from breast cancer, suggesting that RA per se could have a negative effect on cancer outcome (Fig. 2b). This finding is consistent with those of prior studies showing that patients with RA have a higher cancer-associated mortality than the general population [33-35]. As both RA and breast cancer are diseases that predominantly affect women, changes in female hormone levels might increase the aggressiveness of breast cancer in RA patients [36]. A common defect in the immune response may lead to both autoimmunity and impaired cancer surveillance. Ultimately, the mechanisms by which each $\mathrm{RD}$ influences cancer physiology require further investigation. As of now, given the worse prognosis for RD patients with particular cancers, more aggressive cancer treatment tailored to the underlying $\mathrm{RD}$ should be the subject of future studies.

The electronic medical records held at our institution allow us to obtain reliable information regarding the demographic and clinical characteristics of the both cancer and RD patients. In addition, the National Death Register enables us to ascertain the survival status of all enrolled patients. However, the study still has several limitations. First, the relatively small cohort size results in estimates with wide CI. Second, the current study design does not allow estimation of the effects of RD therapy on cancer outcome of patients with corresponding $\mathrm{RD}$ due to the tight collinearity between RD and RD treatment. This is of particular interest, since the treatment of RD focuses on the immune response, which obviously plays a crucial in anti-tumor immunity. Third, 
since cancer treatments vary, often at the discretion of the treating physician, it was difficult to thoroughly analyze the effects of cancer treatment on the survival due to relatively small number of the patients. However, we found that cancer patients with RD and those without RD were treated with comparable aggressiveness except for DM/PM patients with lung cancer; none (0\%) of the 6 lung cancer patients with DM/PM underwent a surgery as compared to the $58.6 \%$ in the lung cancer patients without RD (Additional file 1: Table S2). These limitations should be addressed in a larger prospective study. Last but not the least, the survival analyses were adjusted for Charles comorbidity index and ECOG, although both index were not specifically designed to reflect the functional status of RDs. Retrospective nature of this study did not allow us to collect RD-specific functional status.

\section{Conclusions}

In conclusion, lung and breast cancer patients with RA or DM/PM seemed to be associated with worse survival than non-RD counterparts. Further studies will be needed to address whether cancer treatments tailored to specific RD can improve survival.

\section{Additional file}

Additional file 1: Supplementary material. Table S1. Interval between rheumatic disease and cancer diagnosis in years. Table S2. Cancer treatment according to rheumatic diseases. (DOCX $26 \mathrm{~kb}$ )

\section{Abbreviations}

ACR, American College of Rheumatology; AJCC, American Joint Committee on Cancer; ANOVA, analysis of variance; DM, dermatomyositis; ECOG-PS, Eastern Cooperative Oncology Group performance status; EGD, esophagogastroduodenoscopy; HR, hazard ratio; ILD, interstitial lung disease; NCSP, National Cancer Screening Program; NSAIDs, non-steroidal anti-inflammatory drugs; PM, polymyositis; RA, rheumatoid arthritis; RD, rheumatic diseases; SLE, systemic lupus erythematosus; SSc, systemic sclerosis

\section{Funding}

None.

Availability of data and materials

Data and materials are included in the manuscript.

\section{Authors' contributions}

JKP and JAY participated in the study design, collection and analysis of the data, and preparation of the manuscript. EYA, SHC, YWS and JRC participated in data analysis and preparation of the manuscript. EBL conceived of the study and participated in the study design, interpretation of the data and preparation of the manuscript. All authors read and approved the final manuscript.

\section{Competing interest}

Dr. Eun Bong Lee is a consultant to Pfizer. Dr. Curtis receives support from the Agency for Healthcare Research and Quality (R01HS018517). The authors declare that they have no competing interests.

Consent for publication

Not applicable.

\section{Ethics approval and consent to participate}

The study was approved by the Institutional Review Board of the Seoul National University Hospital. The need for patient consent was waived by the Review Board as the study involved minimal risk and its retrospective nature meant that no identifiable information was used.

\section{Author details}

'Division of Rheumatology, Department of Internal Medicine, Seoul National University College of Medicine, 101, Daehak-ro, Jongno-gu, Seoul 03080, Korea. ${ }^{2}$ Division of Rheumatology, Department of Internal Medicine, Soonchunhyang University Cheonan Hospital, Cheonan, South Korea.

${ }^{3}$ Division of Clinical Immunology \& Rheumatology, University of Alabama at Birmingham, Birmingham, AL, USA.

Received: 11 January 2016 Accepted: 20 June 2016

Published online: 04 July 2016

\section{References}

1. Franks AL, Slansky JE. Multiple associations between a broad spectrum of autoimmune diseases, chronic inflammatory diseases and cancer. Anticancer Res. 2012;32(4):1119-36.

2. Vajdic CM, van Leeuwen MT. Cancer incidence and risk factors after solid organ transplantation. Int J Cancer. 2009;125(8):1747-54.

3. Turesson C, Matteson EL. Malignancy as a comorbidity in rheumatic diseases. Rheumatology (Oxford). 2013;52(1):5-14.

4. Swann JB, Smyth MJ. Immune surveillance of tumors. J Clin Invest. 2007; 117(5):1137-46.

5. Wakabayashi K. NSAIDs as cancer preventive agents. Asian Pac J Cancer Prev. 2000;1(2):97-113.

6. Wu CY, Wu MS, Kuo KN, Wang CB, Chen YJ, Lin JT. Effective reduction of gastric cancer risk with regular use of nonsteroidal anti-inflammatory drugs in Helicobacter pylori-infected patients. J Clin Oncol. 2010;28(18):2952-7.

7. Hemminki K, Liu X, Ji J, Sundquist J, Sundquist K. Effect of autoimmune diseases on mortality and survival in subsequent digestive tract cancers. Ann Oncol. 2012;23(8):2179-84.

8. Rothwell PM, Fowkes FG, Belch JF, Ogawa H, Warlow CP, Meade TW. Effect of daily aspirin on long-term risk of death due to cancer: analysis of individual patient data from randomised trials. Lancet. 2011;377(9759):31-41.

9. Bernatsky S, Ramsey-Goldman R, Clarke A. Malignancy and autoimmunity. Curr Opin Rheumatol. 2006;18(2):129-34.

10. Chang SH, Park JK, Lee YJ, Yang JA, Lee EY, Song YW, Lee EB. Comparison of cancer incidence among patients with rheumatic disease: a retrospective cohort study. Arthritis Res Ther. 2014;16(4):428.

11. Park JK, Choi IA, Lee EY, Song YW, Lee EB. Incidence of malignancy in Takayasu arteritis in Korea. Rheumatol Int. 2014;34(4):517-21.

12. Arnett FC, Edworthy SM, Bloch DA, McShane DJ, Fries JF, Cooper NS, Healey LA, Kaplan SR, Liang MH, Luthra HS, et al. The American Rheumatism Association 1987 revised criteria for the classification of rheumatoid arthritis. Arthritis Rheum. 1988;31(3):315-24.

13. Hochberg MC. Updating the American College of Rheumatology revised criteria for the classification of systemic lupus erythematosus. Arthritis Rheum. 1997:40(9):1725.

14. Bohan A, Peter JB. Polymyositis and dermatomyositis (second of two parts). N Engl J Med. 1975;292(8):403-7.

15. Preliminary criteria for the classification of systemic sclerosis (scleroderma). Subcommittee for scleroderma criteria of the American Rheumatism Association Diagnostic and Therapeutic Criteria Committee. Arthritis Rheum 1980;23(5):581-590.

16. Buccheri G, Ferrigno D, Tamburini M. Karnofsky and ECOG performance status scoring in lung cancer: a prospective, longitudinal study of 536 patients from a single institution. Eur J Cancer. 1996; $32 \mathrm{~A}(7): 1135-41$

17. Quan H, Li B, Couris CM, Fushimi K, Graham P, Hider P, Januel JM, Sundararajan V. Updating and validating the Charlson comorbidity index and score for risk adjustment in hospital discharge abstracts using data from 6 countries. Am J Epidemiol. 2011;173(6):676-82.

18. Schett G, Elewaut D, McInnes IB, Dayer JM, Neurath MF. How cytokine networks fuel inflammation: toward a cytokine-based disease taxonomy. Nat Med. 2013;19(7):822-4.

19. Ho YY, Lagares D, Tager AM, Kapoor M. Fibrosis-a lethal component of systemic sclerosis. Nat Rev Rheumatol. 2014;10(7):390-402. 
20. Sakkas LI, Chikanza IC, Platsoucas CD. Mechanisms of disease: the role of immune cells in the pathogenesis of systemic sclerosis. Nat Clin Pract Rheumatol. 2006;2(12):679-85.

21. Deng GM, Tsokos GC. Pathogenesis and targeted treatment of skin injury in SLE. Nat Rev Rheumatol. 2015;11(11):663-9.

22. Sorensen JB, Klee M, Palshof T, Hansen HH. Performance status assessment in cancer patients. An inter-observer variability study. Br J Cancer. 1993;67(4):773-5.

23. Suh M, Choi KS, Lee YY, Jun JK. Trends in cancer screening rates among Korean Men and women: results from the Korean National Cancer Screening Survey, 2004-2012. Cancer Res Treat. 2013;45(2):86-94.

24. Shin A, Kim J, Park S. Gastric cancer epidemiology in Korea. J Gastric Cancer. 2011;11(3):135-40.

25. Choi KS, Jun JK, Suh M, Park B, Noh DK, Song SH, Jung KW, Lee HY, Choi IJ, Park EC. Effect of endoscopy screening on stage at gastric cancer diagnosis: results of the National Cancer Screening Programme in Korea. Br J Cancer. 2015;112(3):608-12.

26. Adzic TN, Pesut DP, Nagorni-Obradovic LM, Stojsic JM, Vasiljevic MD, Bouros D. Clinical features of lung cancer in patients with connective tissue diseases: a 10-year hospital based study. Respir Med. 2008;102(4):620-4.

27. Wells $\mathrm{AU}$, Denton CP. Interstitial lung disease in connective tissue diseasemechanisms and management. Nat Rev Rheumatol. 2014;10(12):728-39.

28. Olson AL, Swigris JJ, Sprunger DB, Fischer A, Fernandez-Perez ER, Solomon J, Murphy J, Cohen M, Raghu G, Brown KK. Rheumatoid arthritis-interstitial lung disease-associated mortality. Am J Respir Crit Care Med. 2011;183(3):372-8.

29. Chen D, Yuan S, Wu X, Li H, Qiu Q, Zhan Z, Ye Y, Lian F, Liang L, Xu H, et al. Incidence and predictive factors for malignancies with dermatomyositis: a cohort from southern China. Clin Exp Rheumatol. 2014;32(5):615-21.

30. Vesely MD, Kershaw MH, Schreiber RD, Smyth MJ. Natural innate and adaptive immunity to cancer. Annu Rev Immunol. 2011;29:235-71.

31. Hanahan D, Weinberg RA. Hallmarks of cancer: the next generation. Cell. 2011;144(5):646-74.

32. Kuo CF, See LC, Yu KH, Chou IJ, Chang HC, Chiou MJ, Luo SF. Incidence, cancer risk and mortality of dermatomyositis and polymyositis in Taiwan: a nationwide population study. Br J Dermatol. 2011;165(6):1273-9.

33. Hemminki K, Liu X, Ji J, Forsti A, Sundquist J, Sundquist K. Effect of autoimmune diseases on risk and survival in female cancers. Gynecol Oncol. 2012;127(1):180-5.

34. Hemminki K, Li X, Sundquist K, Sundquist J. Cancer risk in hospitalized rheumatoid arthritis patients. Rheumatology (Oxford). 2008;47(5):698-701.

35. Ji J, Liu X, Sundquist K, Sundquist J. Survival of cancer in patients with rheumatoid arthritis: a follow-up study in Sweden of patients hospitalized with rheumatoid arthritis 1 year before diagnosis of cancer. Rheumatology (Oxford). 2011;50(8):1513-8

36. Cutolo M, Sulli A, Straub RH. Estrogen metabolism and autoimmunity. Autoimmun Rev. 2012;11(6-7):A460-4.

\section{Submit your next manuscript to BioMed Central and we will help you at every step:}

- We accept pre-submission inquiries

- Our selector tool helps you to find the most relevant journal

- We provide round the clock customer support

- Convenient online submission

- Thorough peer review

- Inclusion in PubMed and all major indexing services

- Maximum visibility for your research

Submit your manuscript at www.biomedcentral.com/submit

) Biomed Central 